\title{
Co-Culture Strategy of Lactobacillus kefiranofaciens HL1 for Developing Functional Fermented Milk
}

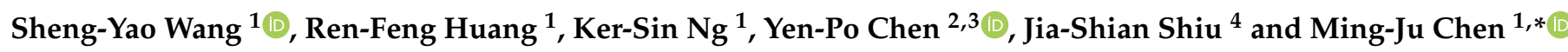 \\ 1 Department of Animal Science and Technology, National Taiwan University, Taipei 10617, Taiwan; \\ yaoyao@ntu.edu.tw (S.-Y.W.); xd2592008@gmail.com (R.-F.H.); r04626001@g.ntu.edu.tw (K.-S.N.) \\ 2 Department of Animal Science, National Chung Hsing University, Taichung 40227, Taiwan; \\ chenyp@dragon.nchu.edu.tw \\ 3 The iEGG and Animal Biotechnology Center, National Chung Hsing University, Taichung 40227, Taiwan \\ 4 Hengchun Branch, Livestock Research Institute, Council of Agriculture, Executive Yuan, \\ Pingtung 94644, Taiwan; Wadeshiu@mail.tlri.gov.tw \\ * Correspondence: cmj@ntu.edu.tw; Tel.: +886-2-33664169
}

Citation: Wang, S.-Y.; Huang, R.-F.; Ng, K.-S.; Chen, Y.-P.; Shiu, J.-S.; Chen, M.J. Co-Culture Strategy of Lactobacillus kefiranofaciens HL1 for Developing Functional Fermented Milk. Foods 2021, 10, 2098. https:// doi.org/10.3390/foods10092098

Academic Editors: Sandra Abreu and Abderrahmane Ait Kaddour

Received: 27 July 2021

Accepted: 2 September 2021

Published: 5 September 2021

Publisher's Note: MDPI stays neutral with regard to jurisdictional claims in published maps and institutional affiliations.

Copyright: (c) 2021 by the authors. Licensee MDPI, Basel, Switzerland. This article is an open access article distributed under the terms and conditions of the Creative Commons Attribution (CC BY) license (https:// creativecommons.org/licenses/by/ $4.0 /)$.

\begin{abstract}
Our previous studies indicated that Lactobacillus kefiranofaciens HL1, isolated from kefir grain, has strong antioxidant activities and anti-aging effects. However, this strain is difficult to use in isolation when manufacturing fermented products due to poor viability in milk. Thus, the purpose of this study was to apply a co-culture strategy to develop a novel probiotic fermented milk rich in L. kefiranofaciens HL1. Each of four selected starter cultures was co-cultured with kefir strain HL1 in different media to evaluate their effects on microbial activity and availability of milk fermentation. The results of a colony size test on de Man, Rogosa and Sharpe (MRS) agar agar, microbial viability, and acidification performance in MRS broth and skimmed milk suggested that Lactococcus lactis subsp. cremoris APL15 is a suitable candidate for co-culturing with HL1. We then co-cultured HL1 and APL15 in skimmed milk and report remarkable improvement in fermentation ability and no negative impact on the viability of strain HL1 or textural and rheological properties of the milk. Through a co-culture strategy, we have improved the viability of kefir strain HL1 in fermented skimmed milk products and successfully developed a novel milk product with a unique flavor and sufficient probiotics.
\end{abstract}

Keywords: Lactobacillus kefiranofaciens; probiotics; co-culture; fermented skimmed milk

\section{Introduction}

Fermented dairy products have been recognized as healthy foods for thousands of years. It is well-known that the fermentation process can extend the shelf-life of fresh milk and nourish the flavor of the final product with high nutrition value. For industrial commercialization, two starter cultures, Lactobacillus delbrueckii subsp. bulgaricus (L. delbrueckii subsp. bulgaricus, LB) and Streptococcus thermophilus (S. thermophilus, ST), are commonly used to produce fermented milk [1], defined as "yogurt" [2]. Other lactic acid bacteria (LAB), such as Lactobacillus (L. acidophilus, L. johnsonii, L. reuteri, and L. rhamnosus) and Bifidobacterium (Bifidobacterium bifidum, B. breve, B. infantis, and B. longum), are also introduced to increase the variety and health benefits of dairy products $[3,4]$.

However, the use of LAB as probiotics in the development of high-quality fermented milk is a challenging task. One of the crucial requirements is to maintain a sufficient number of probiotic cells throughout the manufacturing process and shelf life with no adverse effects on the flavor, aroma, and post-acidification of the final products [5]. Many studies have indicated that the survival of L. acidophilus and genus Bifidobacterium were decreased in fermented milk due to the accumulation of organic acids and hydrogen peroxide [6,7]. Besides, the fermentation characteristics of probiotics in milk, such as acidification time, appropriate taste and aromatic profiles, and tolerance to food additives, should also be considered [3,8,9]. 
Nevertheless, co-culture strategies might provide solutions to tackle the challenges for the utilization of probiotic strains in fermented dairy products. The communications between Lactobacillus and Saccharomyces cerevisiaei through metabolites, microbial aggregation, and biofilm formation could increase microbial counts and organic acids in final products, prevent the contamination from spoilage bacteria during fermentation, and enhance exopolysaccharide production [10]. Recently, Xu et al. [11] thoroughly reviewed the coexistence-relevant mechanisms and molecular regulatory network when co-culturing Lactobacillus with S. cerevisiae in various fermented products. In addition to co-culture strategies between lactobacillus and yeast, fermentation of yogurt starter cultures with L. plantarum could enhance the consumption of sugar in milk for developing acceptable low-sugar yogurt [12]. Casarotti et al. [13] found that acidification rates of B. animalis subsp. lactis and L. acidophilus were improved by $32 \%$ and $74 \%$, respectively, when cocultured with S. thermophilus (ST) in reconstituted milk. The presence of ST also increased the viable cells of L. acidophilus after 28 days of storage [13] and inhibited the acetic acid production of $B$. animalis subsp. lactis to avoid the unpleasant vinegar-like flavor in the dairy product [14]. In another study, co-culturing ST with B. lactis could increase the biomass of the latter by about $38 \%$ and enhance the diacetyl compound in milk as compared with pure culture [15]. However, Ranadheera et al. [16] indicated that different co-culturing combinations of probiotics such as L. acidophilus, B. animalis subsp. Lactis, and Propionibacterium jensenii in goat milk was unable to provide positive effects on the sensory properties due to possible development of unpleasant flavor, organic acid, and unstable curd in fermented goatmilk products during fermentation and storage [17]. Therefore, it is critical to understand the influence of probiotics and starter cultures on the off-flavor of fermented milk by detecting volatile compounds [18] and then select suitable and desirable microorganisms for co-culture strategies. In terms of improving functional properties, ST co-cultured with L. plantarum or B. animalis ssp. lactis could intensify antioxidant capacity and ACE inhibition activity of the fermented milks [19]. Additionally, certain strains of ST as co-cultured with L. brevis could stimulate $L$. brevis to produce the neurotransmitter, $\gamma$-amino-butyric acid (GABA) [20]. Khanlari et al. [21] also demonstrated Enterococcus faecium had a greater acid-producing ability and significantly produced higher amounts of GABA when co-culturing with Lc. lactis subsp. lactis in milk. These findings suggest that a co-culture strategy is applicable to improve the viability, organoleptic characteristics, and functional properties of probiotic microorganisms in dairy products.

Previously, L. kefiranofaciens HL1, an exopolysaccharide producer [22], was isolated from a Taiwanese kefir grain in our lab. This strain has demonstrated in vitro antioxidant activity by measuring the inhibition of linoleic acid peroxidation, chelation ability for $\mathrm{Fe}^{2+}$, and 1,1-diphenyl-2-picrylhydrazyl (DPPH) scavenging activity. The further in vivo study by D-galactose-induced aging mice demonstrated that daily administrating of L. kefiranofaciens HL1 found that the HL1 strain exhibits anti-aging properties by strengthening the resistance to oxidative stress, improving memory and learning abilities, and modulating the composition of gut microbiota [23,24]. However, L. kefiranofaciens HL1 has a low growth rate and poor viability during milk fermentation, which obstructed the usage of this unique strain. Co-culture strategy might provide a solution, but little information is available on the effects of various lactic acid bacteria on L. kefiranofaciens during fermentation. Thus, in the present study, we evaluated the synergistic effects of four selected mesophilic and thermophilic starter strains with L. kefiranofaciens HL1 in milk. The goal of this study was to develop a novel probiotic fermented milk with good fermentation parameters and rheological and sensory properties.

\section{Materials and Methods}

\subsection{Bacteria Cultures}

Lactobacillus kefiranofaciens HL1 (L. kefiranofaciens HL1) and Lactococcus lactis subsp. cremoris APL15 (Lc. lactis subsp. cremoris APL15) were previously isolated from Taiwanese kefir grain and Taiwanese ropy fermented milk, respectively [25-27]. L. delbrueckii subsp. 
bulgaricus BCRC $10696^{\mathrm{T}}$, Streptococcus thermophilus BCRC 12268 (S. thermophilus BCRC 12268), and $S$. thermophilus BCRC $13889^{\mathrm{T}}$ were purchased from Bioresource Collection and Research Center (BCRC) of the Food Industry Research and Development Institute (FIRDI, Hsinchu, Taiwan). All bacteria strains were grown in MRS broth (Acumedia Manufacture, Lansing, MI, USA) at $30{ }^{\circ} \mathrm{C}$ with $1 \%$ inoculation. The cultures were activated twice before further experiments.

\subsection{Screening Candidate Strains for Co-Culture with L. kefiranofaciens HL1 \\ 2.2.1. Colony Size on Agar Plate}

The method was modified as described by Sieuwerts et al. [28]. L. kefiranofaciens HL1 was diluted and spread on MRS agar. The candidate strains were then inoculated at the four corners and in the center of the plate $(2 \mu \mathrm{L} /$ spot $)$. L. delbrueckii subsp. bulgaricus BCRC $10696^{\mathrm{T}}$, S. thermophilus BCRC 12268, S. thermophilus BCRC $13889^{\mathrm{T}}$, and Lc. lactis subsp. cremoris APL15 were selected as candidate strains. Sterilized $0.85 \%$ saline solution was used as the control. After anaerobic cultivation at $30{ }^{\circ} \mathrm{C}$ for $72 \mathrm{~h}$, the plate with the appropriate dilution of L. kefiranofaciens HL1 (20-40 colonies) was photographed, and the average size of colonies was calculated by Image J. The result was presented as relative colony size in percentage by using control as a baseline.

\subsubsection{Cultivation with Supernatant of Candidate Strain}

For the candidate strain that decreased the relative colony size of L. kefiranofaciens HL1, the effect of its supernatant on the growth of L. kefiranofaciens HL1 was evaluated. First, activated culture was centrifuged at $1000 \times g$, for $10 \mathrm{~min}$, and the supernatant was collected and filtered (Millex-GV Filter, $0.22 \mu \mathrm{m}$, Merck KGaA, Darmstadt, Germany). Ten percent of filtered supernatant was added to MRS broth and inoculated with $1 \%$ of L. kefiranofaciens HL1. After cultivation at $30{ }^{\circ} \mathrm{C}$ for $24 \mathrm{~h}, 1 \mathrm{~mL}$ of culture broth was serially diluted with $0.85 \%$ saline solution and plated on MRS agar. The colonies were counted and expressed as colony-forming per unit $(\mathrm{CFU} / \mathrm{mL})$ after incubation at $30^{\circ} \mathrm{C}$ for $24 \mathrm{~h}$.

\subsection{Co-Culture Conditions in MRS Broth and Skimmed Milk}

Each candidate strain ( $1 \%$ inoculation, $\left.\sim 10^{6} \mathrm{CFU} / \mathrm{mL}\right)$ was co-cultured with L. kefiranofaciens HL1 (1\% inoculation, $10^{6} \mathrm{CFU} / \mathrm{mL}$ ) in MRS broth and $10 \%(\mathrm{~g} / \mathrm{mL})$ skimmed milk, separately, at $30{ }^{\circ} \mathrm{C}$ for $24 \mathrm{~h}$. Microbial count of L. kefiranofaciens HL1 was counted with acidified MRS agar ( $\mathrm{pH}$ 5.2, adjusted with $1 \mathrm{~N}$ hydrochloric acid) after anaerobic incubation at $30^{\circ} \mathrm{C}$ for $72 \mathrm{~h}$, while the other strains were calculated with M17 agar (Acumedia Manufacture, Lansing, MI, USA) after aerobic incubation at $30^{\circ} \mathrm{C}$ for $24 \mathrm{~h}$. Besides, the $\mathrm{pH}$ value of cultivated broth was also evaluated with a Lab $850 \mathrm{pH}$ meter (SI Analytics $\mathrm{GmbH}$, Berlin, Germany).

\subsection{Production of Fermented Milk}

L. kefiranofaciens HL1 and selected candidate strains were inoculated in $10 \%(\mathrm{~g} / \mathrm{mL})$ skimmed milk (each at $1 \%, \sim 10^{6} \mathrm{CFU} / \mathrm{mL}$ ), and incubated at $30^{\circ} \mathrm{C}$ for preparing fermented milk samples. Commercial yogurt was made by skimmed milk fermented with commercial starter culture YC-380 (a combination of L. delbrueckii subsp. bulgaricus and S. thermophilus; Chr. Hansen Holding A/S, Hoersholm, Denmark). GDL-induced curd was produced using $1.5 \%$ glucono delta-lactone (GDL). When the $\mathrm{pH}$ values of all experimental groups were below $4.50 \pm 0.05$, fermentation and acidification processes were stopped. The microbial assessment was carried out as previously described. After fermentation and acidification, the samples were stored at $4^{\circ} \mathrm{C}$ for $24 \mathrm{~h}$ for further syneresis, textural, and rheological analyses.

\subsubsection{Physicochemical Properties}

The acidity of fermented milk was determined based on ISO 6901:2010, using $0.5 \mathrm{~mL}$ of $1 \%(\mathrm{~g} / \mathrm{mL}$ in absolute alcohol) phenolphthalein as an indicator for titration. Syneresis was 
performed according to Mani-López et al. [29]. The textural analysis was determined by TA.XT plus Texture Analyser (Stable Micro Systems, Surrey, UK) with a $5 \mathrm{~kg}$ load cell and an A/BE back extrusion cell at the following settings: test speed, $1 \mathrm{~mm} / \mathrm{s}$; post-test speed, $1 \mathrm{~mm} / \mathrm{s}$; distance, $25 \mathrm{~mm}$; and the rate for data acquisition, 200 points per s (pps) [30]. The firmness, consistency, cohesiveness, and viscosity index of fermented milk samples were calculated by positive peak force, area of positive region, peak negative force, and area of negative region, respectively. For viscosity, the tested samples were gently stirred 20 times and set still for $5 \mathrm{~min}$ to allow rebuilding gel structure [31]. RST-CPS Touch Rheometer (Brookfield Engineering Laboratories Inc., MA, USA) with a spindle of RPT-50 (using parallel geometry at $1 \mathrm{~mm}$ gap) was used to record the apparent viscosity of the fermented milk samples for $300 \mathrm{~s}$ under constant rotation speed of $30 \mathrm{rpm}$ at $4{ }^{\circ} \mathrm{C}$ [32].

\subsubsection{Sensory Evaluation}

Sensory evaluation was conducted by performing a hedonic scale test on $30 \mathrm{~mL}$ of fermented milk products $\left(4^{\circ} \mathrm{C}\right)$ from each treated sample. The evaluation was carried out by 30 semi-trained panelists comprised of students and faculty members in the Department of Animal Science and Technology at the National Taiwan University, who were familiar with dairy products. A total of 17 men (56.67\%) and 13 women $(43.33 \%)$ with age between 20 to 31 years took part in this event. Three kinds of fermented milk samples were stored at $4{ }^{\circ} \mathrm{C}$ for $24 \mathrm{~h}$ and were placed in $50 \mathrm{~mL}$ plastic cups coded individually with random three-digit numbers. The samples were tested in a random order and all evaluations were performed at room temperature. Nine-level hedonic tests (1, dislike extremely; 2 , dislike very much; 3 , dislike; 4 , dislike slightly; 5 , neither dislike nor like; 6 , like moderately; 7 , like; 8 , like very much; 9 , like extremely) in terms of appearance, aroma, texture, flavor, and overall evaluation were completed by the panelists [33].

\subsection{Statistical Analysis}

Sensory evaluation was accessed with non-parametric statistics methods, including the Kruskal-Wallis test and Dunn's test, while the other experiments (three replicates) were analyzed using the ANOVA GLM procedure in Statistical Analysis Systems (SAS) software. Comparisons between two groups and multiple groups were processed with student's $t$-test and Tukey's HSD (honest significant difference) test, respectively.

\section{Results and Discussion}

\subsection{Pre-Screening Suitable Starter Cultures for Co-Culturing with Probiotic HL1}

To select a suitable bacteria strain for co-culturing with L. kefiranofaciens HL1 to produce probiotic fermented milk, we first evaluated the effects of four LAB strains often applied as starter cultures in dairy products on the growth of L. kefiranofaciens HL1 incubated in MRS medium. We found that (Figure 1a) the total colony areas of L. kefiranofaciens HL1 solely grown on MRS agar plate (control group) were not significantly different from those co-cultured with S. thermophilus BCRC 12268 (ST 12268), S. thermophilus BCRC $13869^{\mathrm{T}}$ (ST 13869), and Lc. lactis subsp. cremoris APL15. These three strains also showed no influence on the colony-forming rate of L. kefiranofaciens HL1 during incubation, indicating their practicability for co-culturing with L. kefiranofaciens HL1. In contrast, the growth of L. kefiranofaciens HL1 was suppressed by the presence of L. delbrueckii subsp. bulgaricus BCRC $10696^{\mathrm{T}}$ (LB 10696) with a significant reduction in the relative colony size of L. kefiranofaciens HL1 on the MRS plate compared with the control group $(p<0.05)$. To verify the inhibitory phenomenon, L. kefiranofaciens HL1 was inoculated in MRS broth with the additional 10\% filtered supernatant from cultures of LB 10696 (Figure 1b). The viable bacterial counts of $L$. kefiranofaciens HL1 in MRS broth with 10\% filtered supernatant from cultures of LB 10696 were significantly lower during 12 or $24 \mathrm{~h}$ of incubation as compared with the non-supernatant counterpart $(p<0.05)$. Whereas no adverse effect on the cell counts with additional $10 \%$ filtered supernatants from the other three cultures was observed (data not shown). Although LB 10696 was isolated from Bulgarian yogurt 
and is widely used in dairy fermentation to produce commercial yogurt and cheese, their metabolites in supernatant might cause an adverse effect on the viabilities and colony size of L. kefiranofaciens HL1. Our findings were paralleled with previous studies reporting that the activity and viable counts of probiotics belonging to genus Lactobacillus in mix-culture fermented products are decreased by certain L. delbrueckii subsp. bulgaricus strains through nutritional competition and the mutual inhibition of metabolites such as peroxides and organic acid [7,20,29]. Since LB 10696 inhibited the growth of L. kefiranofaciens HL1, this strain was deleted from candidate starters in this study.

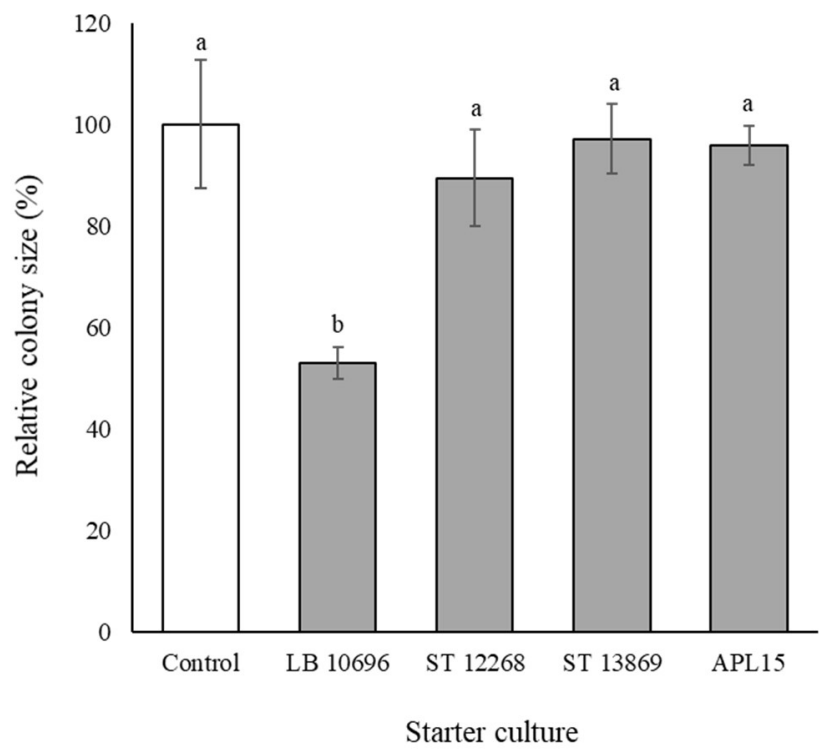

(a)

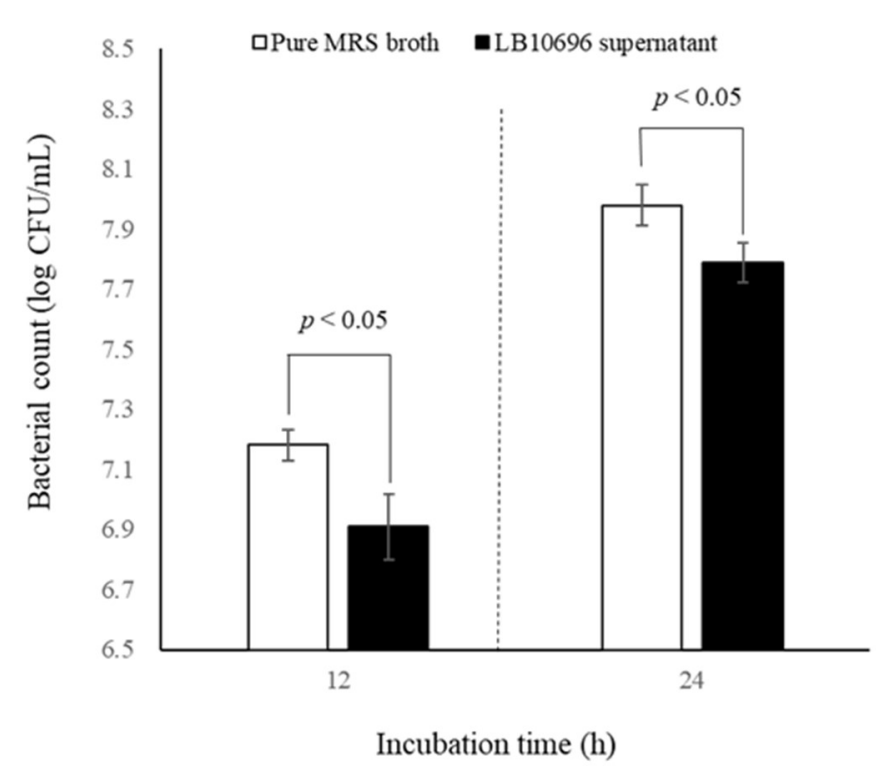

(b)

Figure 1. Effect of starter cultures on the growth of L. kefiranofaciens HL1 incubated in MRS medium. (a) Relative colony size of L. kefiranofaciens HL1 when culturing alone (control) and co-culturing with different starter cultures on MRS agar at $30{ }^{\circ} \mathrm{C}$ for $72 \mathrm{~h}$. Data are given as mean $\pm \mathrm{SD}(n=3)$. a,b bars without the common letter are significantly different $(p<0.05)$. (b) Viable bacterial count of L. kefiranofaciens HL1 in pure MRS broth and in MRS broth with 10\% filtered supernatant from cultures of LB 10696 at $30{ }^{\circ} \mathrm{C}$ for 12 and $24 \mathrm{~h}$. Data are given as mean $\pm \mathrm{SD}(n=3)$. Abbreviations: LB 10696, L. delbrueckii subsp. bulgaricus BCRC 10696T; ST 12268, S. thermophilus BCRC 12268; ST 13869, S. thermophilus BCRC 13869T; APL15, Lc. lactis subsp. cremoris APL15.

Another three candidate starter cultures, including ST 12268, ST 13869, and Lc. lactis subsp. cremoris APL15 (APL15), were further studied in symbiosis with L. kefiranofaciens HL1 in MRS medium. For the viabilities of L. kefiranofaciens HL1 (Figure 2a), no significant change among groups was observed as co-culturing with ST 12268, ST 13869, and APL15 in the MRS at $30^{\circ} \mathrm{C}$ for 12 and $24 \mathrm{~h}$. The viabilities of other starter strains also showed no statistical difference when co-culturing with L. kefiranofaciens HL1 for $24 \mathrm{~h}$ (data not shown).

The acid-producing ability of starter cultures is also important when producing fermented milk products. During 12- and 24-h incubation, the $\mathrm{pH}$ values of the HL1 group in the MRS were $5.86 \pm 0.07$ and $5.14 \pm 0.07$, respectively (Figure 3a). All co-culturing groups had significantly lower $\mathrm{pH}$ compared with the HL1 group $(p<0.05)$, except the HL1+ST 12268 group for 12-h incubation. It is worth noting that the HL1+APL15 group showed a significantly lower $\mathrm{pH}$ than the HL1 and APL15 counterparts in MRS broth, indicating that the co-culture strategy for Lc. lactis subsp. cremoris APL15 and L. kefiranofaciens HL1 could provide a positive effect to stimulate each other to produce organic acids. These findings indicated that Lc. lactis subsp. cremoris APL15 might have stimulated probiotic L. kefiranofaciens HL1 to produce organic acids during co-incubation in MRS medium. 


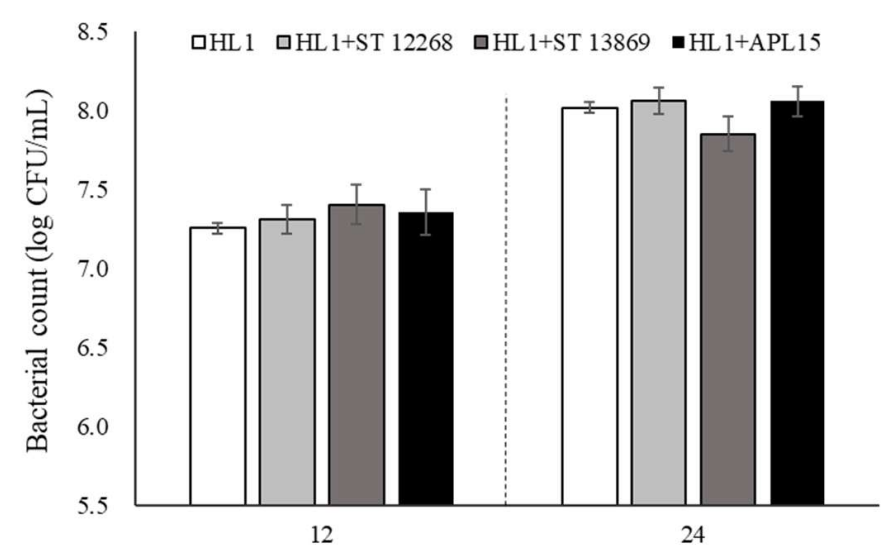

Incubation time $(\mathrm{h})$

(a)

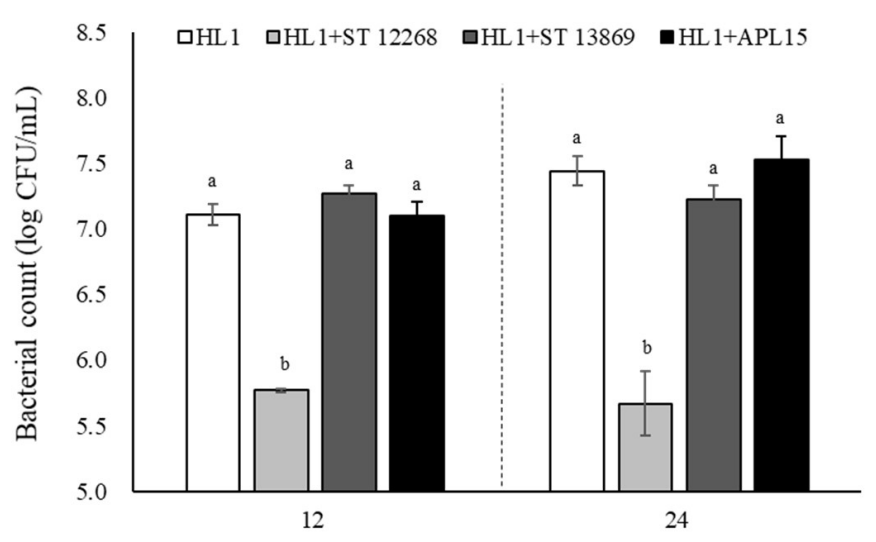

Incubation time (h)

(b)

Figure 2. Effect of HL1 co-culturing with each selected strain on HL1 bacterial counts in MRS broth (a) and skimmed milk (b) at $30{ }^{\circ} \mathrm{C}$ for 12 and $24 \mathrm{~h}$. Data are given as mean $\pm \mathrm{SD}(n=3) .{ }^{\text {a,b }}$ Mean values without the common letter within the same incubation time indicate a significant difference $(p<0.05)$. Abbreviations: HL1, L. kefiranofaciens HL1; ST 12268, S. thermophilus BCRC 12268; ST 13869, S. thermophilus BCRC 13869 ${ }^{\mathrm{T}}$; APL15, Lc. lactis subsp. cremoris APL15.

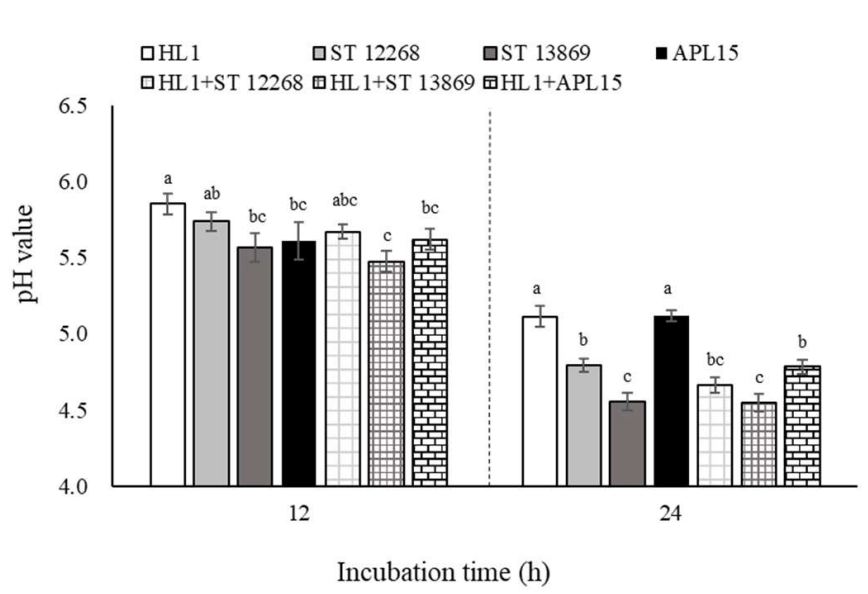

(a)

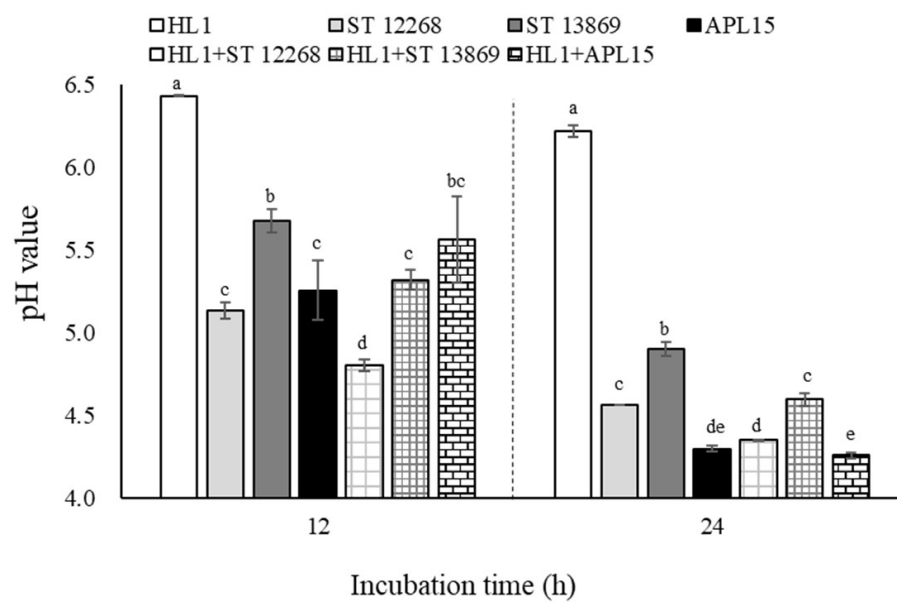

(b)

Figure 3. Effect of HL1 co-culturing with each selected strain on pH values of MRS broth (a) and skimmed milk (b) for both 12- and 24-h fermentation. Data are given as mean $\pm \operatorname{SD}(n=3)$. ${ }^{\text {a-e }}$ Mean values without the common letter within the same incubation time indicate a significant difference $(p<0.05)$. Abbreviations: HL1, L. kefiranofaciens HL1; ST 12268, S. thermophilus BCRC 12268; ST 13869, S. thermophilus BCRC 13869 ${ }^{\mathrm{T}}$; APL15, Lc. lactis subsp. cremoris APL15.

\subsection{Evaluation of Co-culture in Skimmed Milk}

After an investigation using MRS broth, we used skimmed milk to select the coculturing starter cultures. For viability, L. kefiranofaciens HL1 could increase approximately $1.5 \log \mathrm{CFU} / \mathrm{mL}$ after 24-h incubation in skimmed milk (Figure $2 \mathrm{~b}$ ). There were no significant differences in viable bacterial counts among the HL1, HL1+ST 13869, and HL1+APL15 groups. In contrast, the viable bacterial counts of L. kefiranofaciens HL1 were significantly suppressed from $6.0 \log \mathrm{CFU} / \mathrm{mL}$ to $5.5 \log \mathrm{CFU} / \mathrm{mL}$ when co-culturing with ST 12268 in skimmed milk during fermentation $(p<0.05)$. Interestingly, this inhibitory phenomenon was not observed in the MRS medium. Another strain, ST 13869, also showed no negative impact on the viability of L. kefiranofaciens HL1 during co-culture in skimmed milk. Previous studies have demonstrated that different $S$. thermophilus strains show diverse effects on the viability of LAB $[12,15]$. Certain $S$. thermophilus strains could produce specific nutrients, such as formic acid, folic acid, fatty acids, and amino acids during milk fermentation for 
promoting the growth of the Lactobacillus genus [34-36]. Whereas Fontaine and Hols [37] reported that $S$. thermophilus LMD-9 produced bacteriocin-like peptides against Grampositive bacteria and inhibited the growth of nonstarter strains or food-borne pathogen bacteria. The different growth mediums could also influence the production and stability of bacteriocin-like peptides released by some S. thermophilus strains [38]. This finding suggested that the importance for successful application of co-culture strategy is not only dependent on microbial strains; the fermented medium is also a crucial factor. Further studies are required to clarify the inhibitory materials of ST 12268 and relative mechanisms.

Regarding the effect of L. kefiranofaciens HL1 on the bacterial counts of three starter cultures, only Lc. lactis subsp. cremoris APL15 had a significantly lower bacteria count $(p<0.05)$ when co-culturing with L. kefiranofaciens HL1 than that without co-culturing for both 12- and 24-h fermentation (Figure 4). Although both ST strains showed no effect when co-culturing with L. kefiranofaciens HL1, L. kefiranofaciens has been reported to possess an antimicrobial ability [39]. L. kefiranofaciens DD2 could inhibit certain causative bacteria due to suppression of biofilm formation-associated genes, which are related to carbohydrate metabolism, biofilm formation, and adhesion proteins [40]. In fact, Lc. lactis subsp. cremoris APL15 is an exopolysaccharide producer used to increase the ropy and adhesion characteristics of fermented products. The secretion of exopolysaccharides was associated with carbohydrate metabolism and biofilm formation [41]. Thus, the presence of L. kefiranofaciens HL1 in the co-culture system might change the carbohydrate metabolites of Lc. lactis subsp. cremoris and further suppress the bacterial counts.

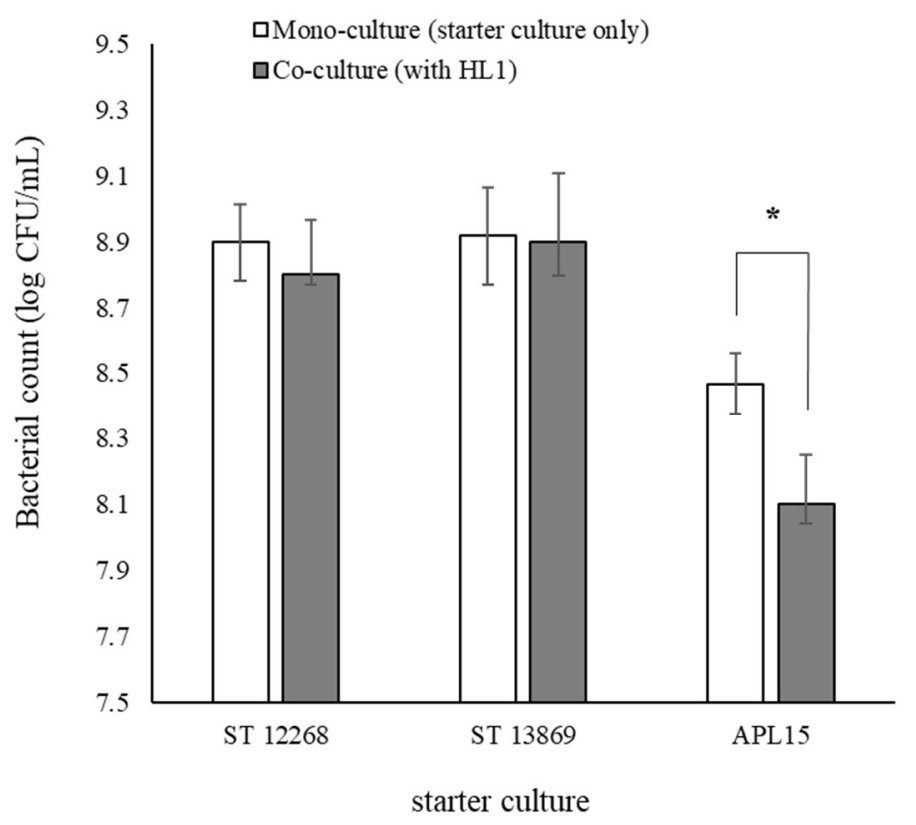

(a)

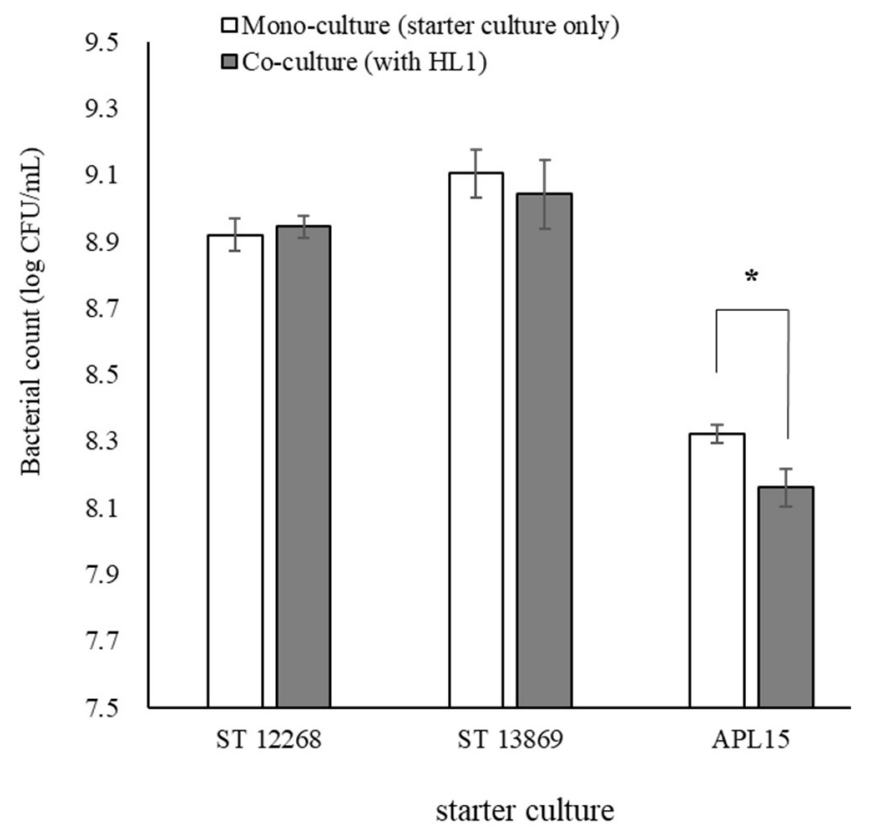

(b)

Figure 4. Effect of HL1 on bacterial counts of each selected co-culturing strain in skimmed milk at $30^{\circ} \mathrm{C}$ for (a) 12 and (b) $24 \mathrm{~h}$. The data are given as mean $\pm \mathrm{SD}(n=3)$. ${ }^{*}$ Bars with a start within the same starter culture indicate a significant difference $(p<0.05)$. Abbreviations: HL1, L. kefiranofaciens HL1; ST 12268, S. thermophilus BCRC 12268; ST 13869, S. thermophilus BCRC 13869T; APL15, Lc. lactis subsp. cremoris APL15.

For acid-producing ability, HL1 co-culturing with ST 12268, ST 13869, and APL 15 could significantly decrease the $\mathrm{pH}$ values of the fermented milk samples for both 12- and 24-h incubations (Figure 3b) $(p<0.05)$. Since L. kefiranofaciens HL1 had a poor acid-producing ability in skimmed milk, the co-culture strategy could help this unique strain to grow and produce acid in fermented milk. In fact, the composition of inoculated bacterial strains highly impacts the acidification of the fermented milk. Sodini et al. [42] demonstrated that some probiotic bacteria grew weakly in milk. This phenomenon was 
accompanied by poor milk acidification. In contrast, the starter strains proliferated quickly and had a positive effect on acidification during milk fermentation. Thus, a co-culture strategy with starter cultures could provide a good opportunity for the commercial production of probiotic fermented milk. Among three starter strains in our study, Lc. lactis subsp. cremoris APL15 presented the highest acid-production. Lc. lactis subsp. cremoris was also a potential probiotic strain due to its exopolysaccharides-producing characteristic and health benefits [43-45]. The findings suggested that Lc. lactis subsp. cremoris APL15 was the best candidate starter strain for co-culturing with L. kefiranofaciens HL1 to develop multiple functional fermented milks. Therefore, we applied a co-culture strategy to develop a fermented milk product with HL1 and APL15 and determine its microbial, physicochemical, and sensory properties.

\subsection{Physicochemical and Sensory Properties of Fermented Milk with HL1}

\subsection{1. $\mathrm{pH}$ Value and Titratable Acidity}

The results of $\mathrm{pH}$ profiles during milk fermentation at $30^{\circ} \mathrm{C}$ (Figure 5a) showed that fermented skimmed milk prepared by culturing with Lc. lactis subsp. cremoris APL15 alone (FSM ${ }^{\mathrm{APL} 15}$ ) and co-culturing L. kefiranofaciens HL1 with Lc. lactis subsp. cremoris APL15 (FSM ${ }^{\mathrm{HL} 1+\mathrm{APL} 15}$ ) had a significantly lower $\mathrm{pH}$ after 12-h fermentation than the HL1 fermented skimmed milk (FSM $\left.{ }^{\mathrm{HL} 1}\right)(p<0.05)$. After 24-h fermentation, FSM HL1+APL15 showed significantly lower $\mathrm{pH}$ and higher titratable acidity than the other two groups (Figure $5 \mathrm{a}, \mathrm{b})(p<0.05)$, and meets the titratable acidity requirement of the Food and Agriculture Organization of the United Nations (FAO) [2] for fermented milk products. Oliveira et al. [46] demonstrated that milk acidification by co-culture of a probiotic strain with a starter culture outperformed the probiotic alone. This might be because the coculture strategy could improve microbial fermentation ability and increase the acid level of products to create a suitable probiotic fermented milk.

\subsubsection{Syneresis and Textural Analysis}

For commercial purposes, it is important to maintain the stability and structure of acid-induced milk curd during shipping and storage. Thus, the syneresis and total textural profiles of the acid-induced milk curds by fermentation or acidification (GDL) were analyzed. Among the four samples, GDL-induced curd showed the highest syneresis $(p<0.05)$ (Table 1). Both FSM ${ }^{\text {APL15 }}$ and FSM ${ }^{\mathrm{HL} 1+\mathrm{APL} 15}$ had lower syneresis compared with the GDL group. As for physical and textural profiles, FSM ${ }^{\mathrm{HL} 1+\mathrm{APL} 15}$ showed a trend to increase firmness, consistency, cohesiveness, and resistance to syneresis as compared with other acid-induced milk curds.

We further measured the rheological properties. The apparent viscosity of four curd samples demonstrated a similar pattern, which decreased with an increase in shear time at a constant temperature and rotation speed (Figure 5c). Both FSM ${ }^{\mathrm{APL} 15}$ and FSM ${ }^{\mathrm{HL} 1+\mathrm{APL} 15}$ had higher apparent viscosity than other groups, even after stirring, which was consistent with our previous results in physical and textural profiles. We noticed that fermented milk made by starter strain of Lc. lactis subsp. cremoris APL15 provided better physical and stable properties than non-fat yogurt or GDL-induced milk curd. Kristo et al. [47] demonstrated that applying the ropy strain of Lc. lactis subsp. cremoris JFR1, when producing fermented milk, could increase the storage modulus and viscosity due to the EPS production. Moreover, the secretion of EPS by Lc. lactis subsp. cremoris JFR1 could reduce the recovery of the protein-protein interaction networks after shearing, and the viscoelastic acid-induced gel would become stir form with ropy stable semisolids. In terms of the dairy industry and consumer market, syneresis or whey separation is an important defect in fermented milk [48]. Our finding suggests that starter strain APL15 might play a crucial role associated with the production of sufficient acid and EPS to form a firm and stable fermented milk product with less syneresis. The EPS produced by Lc. lactis subsp. cremoris APL15 was a great in situ natural stabilizer that prevents syneresis, strengthens the gel structure during processing and storage, and provides sensory properties. 


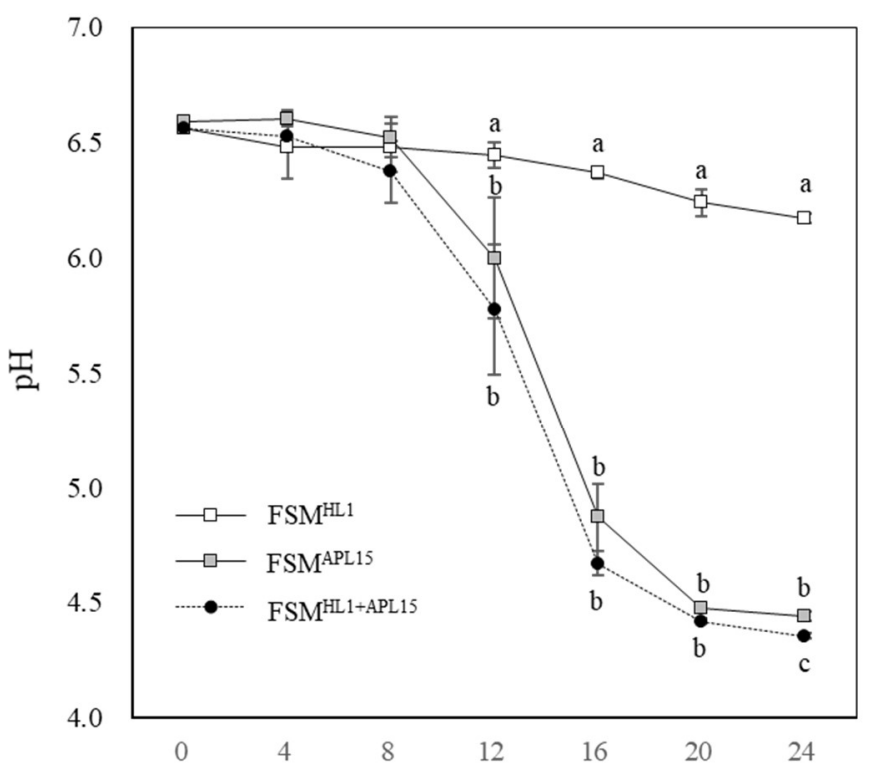

Incubation time $(\mathrm{h})$

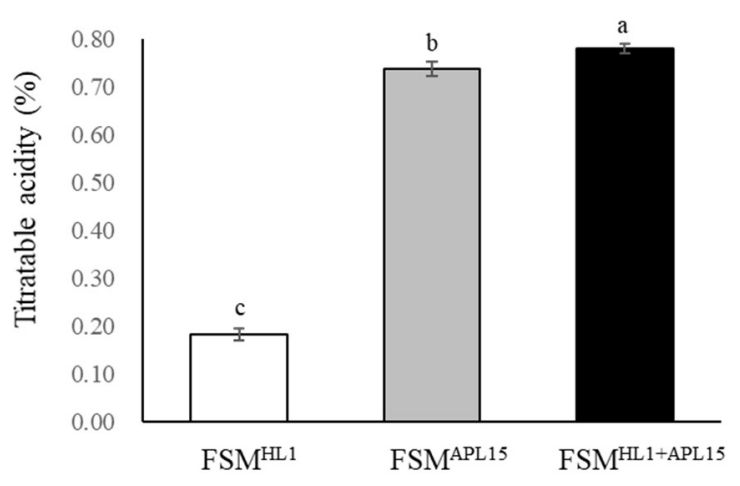

(b)

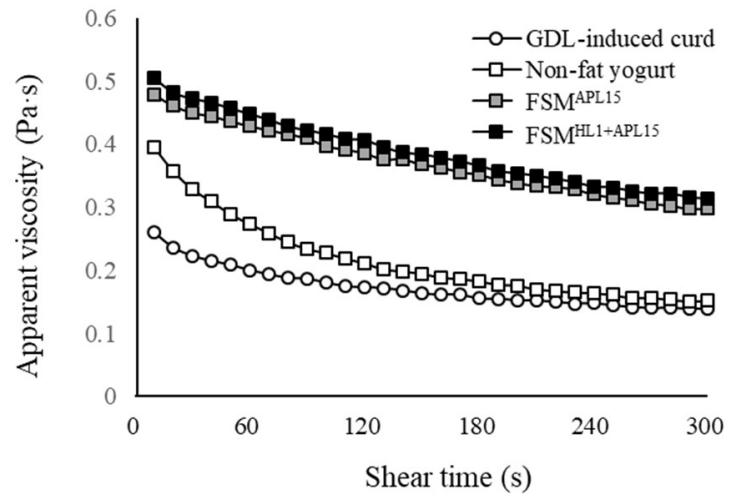

(c)

Figure 5. (a) The $\mathrm{pH}$ profile of fermented skimmed milk inoculated with different cultures during fermentation at $30^{\circ} \mathrm{C}$ for $24 \mathrm{~h}$. (b)Titratable acidity of fermented skimmed milk prepared by three kinds of cultures. The data are given as mean $\pm \mathrm{SD}$ $(n=3) .{ }^{\text {a-c }}$ Points without the common letter within the same incubation time and ${ }^{\mathrm{a}-\mathrm{c}}$ bars without the common letter are significantly different $(p<0.05)$. (c) Apparent viscosity of four kinds of acid-induced milk curds ( $\mathrm{pH} 4.50 \pm 0.05)$ with shear time at constant temperature $\left(4^{\circ} \mathrm{C}\right)$ and rotation speed $(30 \mathrm{rpm})$. FSM ${ }^{\mathrm{HL}}$, fermented skimmed milk prepared by culturing with L. kefiranofaciens HL1 alone; FSM ${ }^{\text {APL15 }}$, fermented skimmed milk prepared by culturing with Lc. lactis subsp.

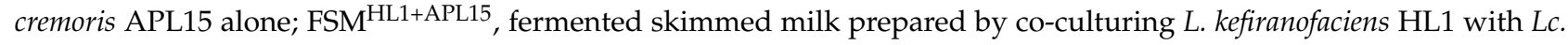
lactis subsp. cremoris APL15; GDL-induced curd, the sample prepared by skimmed milk with 1.5\% GDL; non-fat yogurt: sample prepared by culturing with commercial starter cultures (S. thermophilus and L. delbrueckii subsp. bulgaricus).

Table 1. Syneresis and texture attributes of the fermented skimmed milk samples at $\mathrm{pH} 4.50$.

\begin{tabular}{cccccc}
\hline Samples $^{\mathbf{1}}$ & Syneresis $(\mathbf{\%})$ & Firmness $(\mathbf{g})$ & Consistency $\mathbf{( g} \times \mathbf{s})$ & Cohesiveness $(\mathbf{g})$ & Viscosity Index $(\mathbf{g} \times \mathbf{s})$ \\
\hline GDL-induced curd & $44.89 \pm 10.96^{\mathrm{a}}$ & $15.23 \pm 0.73$ & $389.84 \pm 3.47$ & $8.66 \pm 0.22$ & $45.35 \pm 3.30$ \\
Non-fat yoghurt & $36.59 \pm 2.22^{\mathrm{a}, \mathrm{b}}$ & $15.98 \pm 0.59$ & $395.66 \pm 7.17$ & $8.91 \pm 0.13$ & $50.36 \pm 2.19$ \\
FSM ${ }^{\text {APL15 }}$ & $27.59 \pm 0.88^{\mathrm{b}}$ & $15.63 \pm 0.65$ & $391.05 \pm 5.06$ & $9.52 \pm 0.38$ & $59.91 \pm 8.48$ \\
FSM $^{\text {HL1+APL15 }}$ & $23.40 \pm 2.55^{\mathrm{b}}$ & $17.13 \pm 1.13$ & $402.92 \pm 14.88$ & $9.83 \pm 0.83$ & $54.22 \pm 5.54$ \\
\hline
\end{tabular}

${ }^{1}$ GDL-induced curd: sample prepared by skimmed milk with $1.5 \%$ GDL at $30^{\circ} \mathrm{C}$ to $\mathrm{pH} 4.5$; non-fat yogurt: sample prepared by culturing with commercial starter cultures (S. thermophilus and L. delbrueckii subsp. bulgaricus) at $30^{\circ} \mathrm{C}$ to $\mathrm{pH} 4.5 ; \mathrm{FSM}^{\mathrm{APL} 15}$ : sample prepared by culturing with Lc. lactis subsp. cremoris APL15 alone at $30^{\circ} \mathrm{C}$ to $\mathrm{pH} 4.5 ; \mathrm{FSM}^{\mathrm{HL} 1+\mathrm{APL} 15}$ : sample prepared by co-culturing $L$. kefiranofaciens $\mathrm{HL} 1$ with Lc. lactis subsp. cremoris APL15 at $30^{\circ} \mathrm{C}$ to $\mathrm{pH} 4.5$. Data are given as mean $\pm \mathrm{SD}(n=3)$. Means in the same column with different small letters are significantly different $(p<0.05)$.

\subsubsection{Sensory Evaluation}

To understand how these bacteria affected consumer acceptability and sensory scores of fermented milk, three kinds of fermented milk, including non-fat yogurt, FSM APL15, and

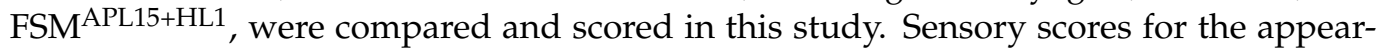
ance of all samples were approximately 6.29-6.82 with no significant difference $(p>0.05)$ (Table 2). The fermented milk produced by inoculation with each culture were uniform 
in appearance since all of them formed intact curds without appearance defects, such as whey separation and coarse surface. The texture scores were also similar and ranged from 5.28 to 5.86 . The sensory analysis of appearance and texture were consistent with the results of texture profiles in Table 2 , and there was no significant difference among the three kinds of fermented milk. However, the scores for aroma, flavor, and overall acceptability of FSM ${ }^{\mathrm{HL} 1+\mathrm{APL} 15}$ were significantly lower than that of other samples. L. kefiranofaciens HL1 participated in milk fermentation would decrease the organoleptic properties of final

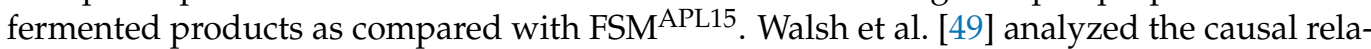
tionship between microbial taxa and volatile compounds in kefir fermentation and found that L. kefiranofaciens correlated with carboxylic acids and ketones associated with cheesy flavors and with esters associated with fruity flavors. Several researchers indicated that certain probiotics would cause unpleasant flavor in products via producing different levels and kinds of metabolic compounds that might decrease the consumer palatability [50-52]. Therefore, it will be necessary to improve the aroma and flavor of FSM $\mathrm{HL}^{\mathrm{H}+\mathrm{APL} 15}$ to increase the total acceptance and consumption. Junaid et al. [53] indicated that probiotic fermented milk produced by adding different flavors, such as strawberry, pineapple, and mango, possessed higher overall acceptability. Moreover, the incorporation of natural fruits or fruit pulps into the probiotic fermented dairy products is an alternative means to improve the sensory profiles [54,55], and would cause our probiotic fermented milk to possess a combination of natural, delicious, and health-promoting properties.

Table 2. Effect of starter cultures on sensory profiles of the fermented skimmed milk samples.

\begin{tabular}{|c|c|c|c|c|c|}
\hline Fermented Skim Milk ${ }^{1}$ & Appearance & Aroma & Texture & Flavor & Overall Acceptability \\
\hline Non-fat yogurt & $6.29 \pm 1.48$ & $6.78 \pm 1.45^{\mathrm{a}}$ & $5.28 \pm 1.96$ & $5.78 \pm 1.80^{\mathrm{a}}$ & $5.88 \pm 1.67^{a}$ \\
\hline FSM ${ }^{\text {APL15 }}$ & $6.82 \pm 1.10$ & $6.05 \pm 1.29 b$ & $5.78 \pm 1.55$ & $5.68 \pm 1.90^{\mathrm{a}}$ & $5.46 \pm 1.78^{\mathrm{a}}$ \\
\hline FSM $^{\text {HL1+APL15 }}$ & $6.72 \pm 1.35$ & $4.66 \pm 1.75^{c}$ & $5.86 \pm 1.45$ & $4.09 \pm 2.21^{b}$ & $4.60 \pm 1.97^{b}$ \\
\hline
\end{tabular}

${ }^{1}$ Non-fat yogurt: sample prepared by culturing with commercial starter cultures (S. thermophilus and L. delbrueckii subsp. bulgaricus) at $30^{\circ} \mathrm{C}$ to $\mathrm{pH} 4.5 ; \mathrm{FSM}^{\mathrm{APL} 15}$ : sample prepared by culturing with Lc. lactis subsp. cremoris APL15 alone; FSM ${ }^{\mathrm{HL} 1+\mathrm{APL} 15}$ : sample prepared by co-culturing L. kefiranofaciens HL1 with Lc. lactis subsp. cremoris APL15 at $30^{\circ} \mathrm{C}$ to $\mathrm{pH} 4.5$. Data are given as mean $\pm \mathrm{SD}(n=30)$. Means in the same column with different superscript letters are significantly different $(p<0.05)$.

\section{Conclusions}

In the present study, Lc. lactis subsp. cremoris APL15 is identified as a superior starter culture for enhancing acid production rate and keeping L. kefiranofaciens HL1 vitality of the fermented ecosystem either in MRS broth or skimmed milk. The co-fermentation of skimmed milk with Lc. lactis subsp. cremoris APL15 and L. kefiranofaciens HL1 for making fermented milk products is a better strategy to provide sufficient probiotic counts and better physicochemical properties with less whey separation. However, improvement of the aroma and flavor of this fermented milk is necessary to increase consumer acceptability. To the best of our knowledge, this is the first report developing a novel probiotic fermented milk using critical LAB isolated from Taiwanese kefir grains and Taiwanese ropy fermented milk.

Author Contributions: Conceptualization, S.-Y.W. and M.-J.C.; methodology, R.-F.H. and K.-S.N.; software, J.-S.S.; validation, Y.-P.C., S.-Y.W., and R.-F.H.; formal analysis, K.-S.N. and J.-S.S.; investigation, S.-Y.W. and Y.-P.C.; resources, M.-J.C. and K.-S.N.; data curation, S.-Y.W.; writing-original draft preparation, S.-Y.W.; writing-review and editing, R.-F.H.; visualization, S.-Y.W.; supervision, M.-J.C., Y.-P.C. and K.-S.N.; project administration, M.-J.C.; funding acquisition, S.-Y.W. and M.-J.C. All authors have read and agreed to the published version of the manuscript.

Funding: This research was funded by the Ministry of Science and Technology, Taiwan (Grant numbers: 109-2313-B-002-017, 110-2313-B-002-056-MY2, and 110-2313-B-002-047-MY3) and the Council of Agriculture, Executive Yuan, Taiwan (Grant number: 109AS-3.1.1-AD-U1).

Institutional Review Board Statement: Not applicable. 
Informed Consent Statement: Not applicable.

Data Availability Statement: The data presented in this article are available on reasonable request, from the corresponding author.

Acknowledgments: We acknowledge the funding of this research by the Ministry of Science and Technology, Taiwan and the Council of Agriculture, Executive Yuan, Taiwan.

Conflicts of Interest: The authors declare no conflict of interest.

\section{References}

1. Hamann, W.T.; Marth, E.H. Survival of Streptococcus thermophilus and Lactobacillus bulgaricus in Commercial and Experimental Yogurts. J. Food Prot. 1984, 47, 781-786. [CrossRef]

2. Codex Alimentarius Commission. Codex-Standard 243-2003: Codex Standard for Fermented Milks (2003). Available online: http://www.fao.org/input/download/standards/400/CXS_243e.pdf (accessed on 18 December 2020).

3. La Torre, L.; Tamime, A.Y.; Muir, D.D. Rheology and sensory profiling of set-type fermented milks made with different commercial probiotic and yoghurt starter cultures. Int. J. Dairy Technol. 2003, 56, 163-170. [CrossRef]

4. Farag, M.A.; El Hawary, E.A.; Elmassry, M. Rediscovering acidophilus milk, its quality characteristics, manufacturing methods, flavor chemistry and nutritional value. Crit. Rev. Food Sci. Nutr. 2019, 60, 3024-3041. [CrossRef]

5. Heller, K.J. Probiotic bacteria in fermented foods: Product characteristics and starter organisms. Am. J. Clin. Nutr. 2001, 73, 374s-379s. [CrossRef] [PubMed]

6. Donkor, O.; Henriksson, A.; Vasiljevic, T.; Shah, N. Effect of acidification on the activity of probiotics in yoghurt during cold storage. Int. Dairy J. 2006, 16, 1181-1189. [CrossRef]

7. Ng, E.W.; Yeung, M.; Tong, P.S. Effects of yogurt starter cultures on the survival of Lactobacillus acidophilus. Int. J. Food Microbiol. 2011, 145, 169-175. [CrossRef] [PubMed]

8. Vinderola, C.; Costa, G.; Regenhardt, S.; Reinheimer, J. Influence of compounds associated with fermented dairy products on the growth of lactic acid starter and probiotic bacteria. Int. Dairy J. 2002, 12, 579-589. [CrossRef]

9. Lucas, A.; Sodini, I.; Monnet, C.; Jolivet, P.; Corrieu, G. Probiotic cell counts and acidification in fermented milks supplemented with milk protein hydrolysates. Int. Dairy J. 2004, 14, 47-53. [CrossRef]

10. Cheirsilp, B.; Shimizu, H.; Shioya, S. Enhanced kefiran production by mixed culture of Lactobacillus kefiranofaciens and Saccharomyces cerevisiae. J. Biotechnol. 2003, 100, 43-53. [CrossRef]

11. Xu, Z.; Lu, Z.; Soteyome, T.; Ye, Y.; Huang, T.; Liu, J.; Harro, J.M.; Kjellerup, B.V.; Peters, B.M. Polymicrobial interaction between Lactobacillus and Saccharomyces cerevisiae: Coexistence-relevant mechanisms. Crit. Rev. Microbiol. 2021, 47, 386-396. [CrossRef]

12. Zhang, S.; Xu, Z.; Qin, L.; Kong, J. Low-sugar yogurt making by the co-cultivation of Lactobacillus plantarum WCFS1 with yogurt starter cultures. J. Dairy Sci. 2020, 103, 3045-3054. [CrossRef]

13. Casarotti, S.; Monteiro, D.A.; Moretti, M.; Penna, A.L.B. Influence of the combination of probiotic cultures during fermentation and storage of fermented milk. Food Res. Int. 2014, 59, 67-75. [CrossRef]

14. Rodrigues, D.; Rocha-Santos, T.A.; Pereira, C.I.; Gomes, A.M.; Malcata, F.X.; Freitas, A.C. The potential effect of FOS and inulin upon probiotic bacterium performance in curdled milk matrices. LWT 2011, 44, 100-108. [CrossRef]

15. Oliveira, R.P.D.S.; Perego, P.; de Oliveira, M.N.; Converti, A. Growth, organic acids profile and sugar metabolism of Bifidobacterium lactis in co-culture with Streptococcus thermophilus: The inulin effect. Food Res. Int. 2012, 48, 21-27. [CrossRef]

16. Ranadheera, C.S.; Evans, C.A.; Adams, M.; Baines, S.K. Co-culturing of probiotics influences the microbial and physico-chemical properties but not sensory quality of fermented dairy drink made from goats' milk. Small Rumin. Res. 2016, 136, 104-108. [CrossRef]

17. Slačanac, V.; Božanić, R.; Hardi, J.; Szabó, J.R.; Lučan, M.; Krstanović, V. Nutritional and therapeutic value of fermented caprine milk. Int. J. Dairy Technol. 2010, 63, 171-189. [CrossRef]

18. Zhang, L.; Mi, S.; Liu, R.; Sang, Y.; Wang, X. Evaluation of Volatile Compounds in Milks Fermented Using Traditional Starter Cultures and Probiotics Based on Odor Activity Value and Chemometric Techniques. Molecules 2020, 25, 1129. [CrossRef]

19. Li, S.; Tang, S.; He, Q.; Hu, J.; Zheng, J. In vitro antioxidant and angiotensin-converting enzyme inhibitory activity of fermented milk with different culture combinations. J. Dairy Sci. 2020, 103, 1120-1130. [CrossRef]

20. Wu, Q.; Law, Y.-S.; Shah, N.P. Dairy Streptococcus thermophilus improves cell viability of Lactobacillus brevis NPS-QW-145 and its $\gamma$-aminobutyric acid biosynthesis ability in milk. Sci. Rep. 2015, 5, 12885. [CrossRef] [PubMed]

21. Khanlari, Z.; Moayedi, A.; Ebrahimi, P.; Khomeiri, M.; Sadeghi, A. Enhancement of gamma-aminobutyric acid (GABA) content in fermented milk by using Enterococcus faecium and Weissella confusa isolated from sourdough. J. Food Process. Preserv. 2021, $45, \mathrm{e} 15869$.

22. Ng, K.-S.; Wang, S.-Y.; Chen, M.-J. A novel immobilized cell system involving Taiwanese kefir microorganisms and sugar cane pieces for fermented milk production. J. Dairy Sci. 2020, 103, 141-149. [CrossRef] [PubMed]

23. Hsieh, Y.T. Selecting Probiotics with Anti-Oxidative Ability and Investigating Their Anti-Aging Effects. Master's Thesis, National Taiwan University, Taipei, Taiwan, 2016. 
24. Ho, S.T.; Hsieh, Y.T.; Chen, M.J. Probiotic strains mixture Pm1 and Lactobacillus kefiranofaciens attenuated D-Galactose-induced oxidative stress and brain damage in mice model. In Proceedings of the 9th Asian Conference on Lactic Acid Bacteria, Gwangju, Korea, 3-5 July 2017.

25. Chen, H.-C.; Wang, S.-Y.; Chen, M.-J. Microbiological study of lactic acid bacteria in kefir grains by culture-dependent and culture-independent methods. Food Microbiol. 2008, 25, 492-501. [CrossRef]

26. Wang, S.-Y.; Chen, K.-N.; Lo, Y.-M.; Chiang, M.-L.; Chen, H.-C.; Liu, J.-R.; Chen, M.-J. Investigation of microorganisms involved in biosynthesis of the kefir grain. Food Microbiol. 2012, 32, 274-285. [CrossRef] [PubMed]

27. Chang, Y.C. Isolation and Identification of Exopolysaccharide-Producing Lactic Acid Bacteria from Taiwanese Ropy Fermented Milk for Low-Fat Fermented Milk Production. Master's Thesis, National Taiwan University, Taipei, Taiwan, 2018.

28. Sieuwerts, S.; Bron, P.A.; Smid, E.J. Mutually stimulating interactions between lactic acid bacteria and Saccharomyces cerevisiae in sourdough fermentation. LWT 2018, 90, 201-206. [CrossRef]

29. Mani-López, E.; Palou, E.; López-Malo, A. Probiotic viability and storage stability of yogurts and fermented milks prepared with several mixtures of lactic acid bacteria. J. Dairy Sci. 2014, 97, 2578-2590. [CrossRef]

30. Brennan, C.S.; Tudorica, C.M. Carbohydrate-based fat replacers in the modification of the rheological, textural and sensory quality of yoghurt: Comparative study of the utilisation of barley beta-glucan, guar gum and inulin. Int. J. Food Sci. Technol. 2008, 43, 824-833. [CrossRef]

31. Purwandari, U.; Shah, N.; Vasiljevic, T. Effects of exopolysaccharide-producing strains of Streptococcus thermophilus on technological and rheological properties of set-type yoghurt. Int. Dairy J. 2007, 17, 1344-1352. [CrossRef]

32. Sah, B.N.P.; Vasiljevic, T.; McKechnie, S.; Donkor, O.N. Physicochemical, textural and rheological properties of probiotic yogurt fortified with fibre-rich pineapple peel powder during refrigerated storage. LWT Food Sci. Technol. 2016, 65, 978-986. [CrossRef]

33. Meilgaard, M.C.; Civille, G.; Carr, B.T. Sensory Evaluation Techniques, 4th ed.; CRC Press: Boca Raton, FL, USA, 2007.

34. Courtin, P.; Monnet, V.; Rul, F. Cell-wall proteinases PrtS and PrtB have a different role in Streptococcus thermophilus / Lactobacillus bulgaricus mixed cultures in milk. Microbiology 2002, 148, 3413-3421. [CrossRef] [PubMed]

35. Savijoki, K.; Ingmer, H.; Varmanen, P. Proteolytic systems of lactic acid bacteria. Appl. Microbiol. Biotechnol. 2006, 71, 394-406. [CrossRef]

36. Sieuwerts, S.; Molenaar, D.; van Hijum, S.A.F.T.; Beerthuyzen, M.; Stevens, M.J.A.; Janssen, P.W.M.; Ingham, C.J.; de Bok, F.A.M.; de Vos, W.M.; van Hylckama Vlieg, J.E.T. Mixed-Culture Transcriptome Analysis Reveals the Molecular Basis of Mixed-Culture Growth in Streptococcus thermophilus and Lactobacillus bulgaricus. Appl. Environ. Microbiol. 2010, 76, 7775-7784. [CrossRef] [PubMed]

37. Fontaine, L.; Hols, P. The inhibitory spectrum of thermophilin 9 from Streptococcus thermophilus LMD-9 depends on the production of multiple peptides and the activity of BlpGSt, a thiol-disulfide oxidase. Appl. Environ. Microbiol. 2007, 74, 1102-1110. [CrossRef]

38. Renye, J.A.; Somkuti, J.A.G.A.; Garabal, J.I.; Steinberg, D.H. Bacteriocin production by Streptococcus thermophilus in complex growth media. Biotechnol. Lett. 2016, 38, 1947-1954. [CrossRef] [PubMed]

39. Santos, A.; Mauro, M.S.; Sanchez, A.; Torres, J.; Marquina, D. The Antimicrobial Properties of Different Strains of Lactobacillus spp. Isolated from Kefir. Syst. Appl. Microbiol. 2003, 26, 434-437. [CrossRef] [PubMed]

40. Jeong, D.; Kim, D.-H.; Song, K.-Y.; Seo, K.-H. Antimicrobial and anti-biofilm activities of Lactobacillus kefiranofaciens DD2 against oral pathogens. J. Oral Microbiol. 2018, 10, 1472985. [CrossRef] [PubMed]

41. Sutherland, I.W. Biofilm exopolysaccharides: A strong and sticky framework. Microbiology 2001, 147, 3-9. [CrossRef]

42. Sodini, I.; Lucas, A.; Oliveira, M.; Remeuf, F.; Corrieu, G. Effect of Milk Base and Starter Culture on Acidification, Texture, and Probiotic Cell Counts in Fermented Milk Processing. J. Dairy Sci. 2002, 85, 2479-2488. [CrossRef]

43. Kitazawa, H.; Yamaguchi, T.; Itoh, T. B-Cell Mitogenic Activity of Slime Products Produced from Slime-Forming, Encapsulated Lactococcus lactis ssp. cremoris. J. Dairy Sci. 1992, 75, 2946-2951. [CrossRef]

44. Nakajima, H.; Suzuki, Y.; Hirota, T. Cholesterol Lowering Activity of Ropy Fermented Milk. J. Food Sci. 1992, 57, 1327-1329. [CrossRef]

45. Ruas-Madiedo, P.; Gueimonde, M.; Reyes-Gavilán, C.D.L.; Salminen, S. Short Communication: Effect of Exopolysaccharide Isolated from "Viili" on the Adhesion of Probiotics and Pathogens to Intestinal Mucus. J. Dairy Sci. 2006, 89, 2355-2358. [CrossRef]

46. Oliveira, R.P.D.S.; Perego, P.; Converti, A.; De Oliveira, M.N. Growth and acidification performance of probiotics in pure culture and co-culture with Streptococcus thermophilus: The effect of inulin. LWT 2009, 42, 1015-1021. [CrossRef]

47. Kristo, E.; Miao, Z.; Corredig, M. The role of exopolysaccharide produced by Lactococcus lactis ssp cremoris in structure formation and recovery of acid milk gels. Int. Dairy J. 2011, 21, 656-662. [CrossRef]

48. Mizrahi, S. Syneresis in food gels and its implications for food quality. In Chemical Deterioration and Physical Instability of Food and Beverages; Skibsted, L.H., Risbo, J., Andersen, M.L., Eds.; Woodhead Publishing: Cambridge, UK, 2010; pp. $324-348$.

49. Walsh, A.M.; Crispie, F.; Kilcawley, K.; O'Sullivan, O.; O'Sullivan, M.G.; Claesson, M.; Cotter, P.D. Microbial Succession and Flavor Production in the Fermented Dairy Beverage Kefir. mSystems 2016, 1, e00052-16. [CrossRef]

50. Tamime, A.Y.; Robinson, R.K. Yoghurt: Science and Technology, 2nd ed.; CRC Press: Boca Raton, FL, USA, 1999.

51. Luckow, T.; Sheehan, V.; Fitzgerald, G.; Delahunty, C. Exposure, health information and flavour-masking strategies for improving the sensory quality of probiotic juice. Appetite 2006, 47, 315-323. [CrossRef]

52. Chen, C.; Zhao, S.; Hao, G.; Yu, H.; Tian, H.; Zhao, G. Role of lactic acid bacteria on the yogurt flavour: A review. Int. J. Food Prop. 2017, 20, S316-S330. [CrossRef] 
53. Junaid, M.; Javed, I.; Abdullah, M.; Gulzar, M.; Younas, U.; Nasir, J.; Ahmad, N. Development and quality assessment of flavored probiotic acidophilus milk. J. Anim. Plant Sci. 2013, 23, 1342-1346.

54. Srisuvor, N.; Chinprahast, N.; Prakitchaiwattana, C.; Subhimaros, S. Effects of inulin and polydextrose on physicochemical and sensory properties of low-fat set yoghurt with probiotic-cultured banana purée. LWT 2013, 51, 30-36. [CrossRef]

55. Gallina, D.A.; Barbosa, P.D.P.M.; Ormenese, R.D.C.S.C.; Garcia, A.D.O. Development and characterization of probiotic fermented smoothie beverage. Rev. Ciência Agronômica 2019, 50, 378-386. [CrossRef] 